\title{
TRAJECTORY ADJUSTMENT OF MOBILE LASER SCAN DATA IN GPS DENIED ENVIRONMENTS
}

\author{
P. Schaer ${ }^{\mathrm{a}, *}$, J. Vallet ${ }^{\mathrm{b}}$ \\ a GEOSAT SA, Route du Manège 59b, 1950 Sion, Switzerland - schaer@geosat.ch \\ ${ }^{\mathrm{b}}$ HELIMAP SYSTEM SA, Le Grand-Chemin 73, 1066 Epalinges, Switzerland -julien.vallet@ helimap.ch
}

KEY WORDS: MLS, mobile mapping, LiDAR, long tunnel survey, GPS denied environment

\begin{abstract}
:
Mobile mapping often occurs in environments with poor or no GNSS signal reception (tunnels, urban canons, canopy ...). Most mobile scanning systems are equipped with tactical grade inertial systems, GNSS outages longer than 30-60 seconds may lead to a rapid decrease in absolute positioning accuracy that might be below the client's expectations. In order to guarantee sufficient positioning accuracy even in such environments the data must be corrected. This can either happen by readjusting the final point cloud using control points (3D translations) or by directly correcting the trajectory by adding external position updates. The approach presented in this paper consists in detecting targets (either existing or placed before measurement) that can easily be identified in the point cloud and that have been measured independently. By identifying the 3D point closest to the target's center, computing the coordinate difference to the corresponding GCP and retrieving its GNSS-timestamp and the internal scanner coordinates, an external position update at a given time can be computed. This procedure has proven its efficiency in several projects including scanning tunnels up to $5 \mathrm{~km}$ length, where the positional error (in 3D) of the resulting point cloud could be reduced from $5 \mathrm{~m}$ (in the middle of the tunnel) down to better than $5 \mathrm{~cm}$ using GCPS only every $400 \mathrm{~m}$.
\end{abstract}

\section{INTRODUCTION}

The acquisition of laser point clouds for road environments by mobile mapping has become very popular, due to the fact that compared to terrestrial static laser scanning (TLS), kinematic acquisition of $3 \mathrm{D}$ data has substantial benefits in terms of cost efficiency (less time spent measuring, no closure of road necessary) and security (no workers exposed to traffic directly). Mobile mapping often occurs in environments where GNSS signals are partly (urban canons, canopy) or completely (tunnels, galleries) masked. Even though most mobile scanning systems are equipped with tactical or navigation grade IMUs, GNSS outages longer than 30-60 seconds may lead to a rapid decrease in absolute positioning accuracy that might be below the client's expectations (see table 1). In order to guarantee sufficient positioning accuracy even in such environments the data must be corrected.

Often these corrections are carried out by measuring control points that are used to create a local $3 \mathrm{D}$ deformation model which is applied to readjust directly the final point cloud generated by direct georeferencing (a posteriori adjustment). However this technique requires the presence of many control points (at least every 50meters), a task that may lead to high costs due to road closure and difficulty in access. As no correction on the trajectory data itself is applied, such procedure also neglects the non-linear behavior of position errors in trajectories derived from inertial data. It is therefore preferable to perform the corrections directly to the trajectory by adding external position updates (a priori adjustment).

In this paper we discuss the methodology for generating such external position updates directly form signalized targets in the point cloud. We also present results from a test in a tunnel of $5 \mathrm{~km}$ length that validate our approach.

\section{PROPOSED APPROACH}

\subsection{Mathematical model}

\begin{tabular}{|c|c|c|c|c|}
\hline IMU Grade & $\begin{array}{c}\text { MEMS } \\
\text { low cost }\end{array}$ & $\begin{array}{l}\text { MEMS } \\
\text { tactical }\end{array}$ & $\begin{array}{c}\text { FOG } \\
\text { tactical }\end{array}$ & $\begin{array}{c}\text { FOG } \\
\text { navigation }\end{array}$ \\
\hline Angular drift & $5-10^{\circ} / \mathrm{h}$ & $1-3 \% / h$ & $0.1-1^{\circ} / \mathrm{h}$ & $<0.01^{\circ} / \mathrm{h}$ \\
\hline $\begin{array}{l}\text { Position drift } \\
\text { after 60sec }\end{array}$ & $1-10 \mathrm{~m}$ & $20-80 \mathrm{~cm}$ & $15-30 \mathrm{~cm}$ & $10-15 \mathrm{~cm}$ \\
\hline $\begin{array}{l}\text { Position } \\
\text { accuracy } \\
\text { GPS/INS }\end{array}$ & $10 \mathrm{~cm}$ & $5-10 \mathrm{~cm}$ & $5 \mathrm{~cm}$ & $5 \mathrm{~cm}$ \\
\hline $\begin{array}{l}\text { Angular } \\
\text { accuracy } \\
\text { GPS/INS }\end{array}$ & $0.1-0.2^{\circ}$ & $0.05^{\circ}$ & $<0.02^{\circ}$ & $<0.005^{\circ}$ \\
\hline Typical usage & Leisure & $\begin{array}{c}\text { Short } \\
\text { range } \\
\text { ALS }\end{array}$ & $\begin{array}{l}\text { ALS, } \\
\text { MLS }\end{array}$ & ALS, MLS \\
\hline Price & $50-1^{\prime} 000 €$ & $\begin{array}{c}5-10 ' 000 \\
€\end{array}$ & $30^{\prime} 000 €$ & $100^{\prime} 000 €$ \\
\hline
\end{tabular}

Table 1: Different types of IMU, their accuracies and usage

Compared to airborne laser scanning, where typical ranges reach from $200 \mathrm{~m}$ up to $3500 \mathrm{~m}$, the measured laser ranges in mobile mapping systems (denoted MLS hereafter) are much shorter (typically $2-50 \mathrm{~m}$ ). In confined environments, such as tunnels, the maximal ranges often do not even exceed $5 \mathrm{~m}$. If the MLS is equipped with an IMU with an angular drift of less than $0.1 \%$, the point cloud error due to the angular drift after one hour of dead reckoning does not exceed $1 \mathrm{~cm}$ and can therefore be neglected for the readjustment of the data. The positional errors however increase much faster and may reach easily decimeter level after only 1 minute of dead reckoning even for navigation grade IMUs (see table 1). Therefore adding external position updates into the Kalman Filter (KF) at regular intervals

\footnotetext{
* Corresponding author
} 
is of uppermost importance in order to guarantee a subdecimeter absolute position accuracy of the computed point cloud.

Now we can consider the direct georeferencing formula (Baltsavias, 1999) that expresses the computation of a point cloud coordinate $\mathbf{p}$ in a mapping frame $\mathrm{m}$ in function of scanner measurements, the GPS/INS measurements and system calibration parameters:

$\mathbf{p}(t)^{m}=\mathbf{s}(t)^{m}+\mathbf{R}_{b}^{m}(t)\left[\mathbf{R}_{s}^{b} \rho(t)\left(\begin{array}{c}\sin \theta(t) \\ 0 \\ \cos \theta(t)\end{array}\right)+\mathbf{a}^{b}\right]$

Where

$\mathbf{p}(t)^{\mathrm{m}}$

$\rho(t), \theta(t)$

$\mathbf{s}(t)^{\mathrm{m}}$

$\mathbf{R}_{\mathrm{b}}{ }^{\mathrm{m}}(\mathrm{r}(t), \mathrm{p}(t), \mathrm{y}(t))$ is the attitude matrix from the IMU body $\mathbf{R}_{\mathrm{s}}^{\mathrm{b}}\left(\mathrm{e}_{\mathrm{x}}, \mathrm{e}_{\mathrm{y}}, \mathrm{e}_{\mathrm{z}}\right)$ frame to the mapping frame at time $t$

$\mathbf{R}_{\mathrm{s}}\left(\mathrm{e}_{\mathrm{x}}, \mathrm{e}_{\mathrm{y}}, \mathrm{e}_{\mathrm{z}}\right)$

$\mathbf{a}^{\mathrm{b}}$

is a point in the mapping frame computed at time $t$

are the scanner range and the encoder angle measured by the scanner at time $t$

is the position of the scanner center in the arbitrary mapping frame $\mathrm{m}$ at time $t$ the boresight matrix describing the angular offsets between the body frame and the scanner frame is the lever-arm offset between the IMU and scanner frame origins expressed in the body frame

In the case a point was measured independently in 3D (denominated $\mathbf{g c p}^{\mathrm{m}}$ ) that materializes a clearly identifiable target, we can find a point $\mathbf{p}\left(t_{i}\right)^{\mathrm{m}}$ in the point cloud generated by direct georeferencing that is closest in $3 \mathrm{D}$ to this point

$\left\|\operatorname{gcp}^{m}-\mathbf{p}\left(t_{i}\right)^{m}\right\|=\min$

Assuming that the boresight $\mathbf{R}_{\mathrm{s}}^{\mathrm{b}}$ and leverarm $\mathbf{a}^{\mathrm{b}}$ are perfectly calibrated, that the attitude angles (rpy) at time $t_{i}$ have been estimated by GPS/INS integration with an accuracy better than $0.1^{\circ}$, the only unknown in equation 1 is the position of the scanner $\mathbf{s}\left(t_{i}\right)^{\mathrm{m}}$ at a given time $t_{i}$. Accordingly equation 1 can be reformulated in order to compute scanner positions at a time $t_{i}$ replacing the $3 \mathrm{D}$ coordinates of $\mathbf{p}\left(t_{i}\right)^{\mathrm{m}}$ with the ones observed by independent control measures $\mathbf{g c p}^{\mathrm{m}}$.

\subsection{Procedure}

Based on the mathematical model explained before the following calibration procedure has been developed (figure 1):

- The trajectory is first computed by GPS/INS integration without any external position updates

- $\quad$ A point cloud (containing intensity value and the precise timestamp for every record) is generated based on this initial trajectory

- Within the initial point cloud the target center coordinates corresponding to a given GCP are extracted

- $\quad$ The timestamp $t_{i}$ to the point closest to the target center is extracted and the 3D difference between the target center in the point cloud and the GCP coordinates is computed in order to compute position updates at the time $t_{i}$
An adjusted trajectory is recomputed including the position updates at the times $t_{1} \ldots t_{n}$

Finally the adjusted trajectory is used to compute the final point cloud where the targets centers within the point cloud match the GCP coordinates.

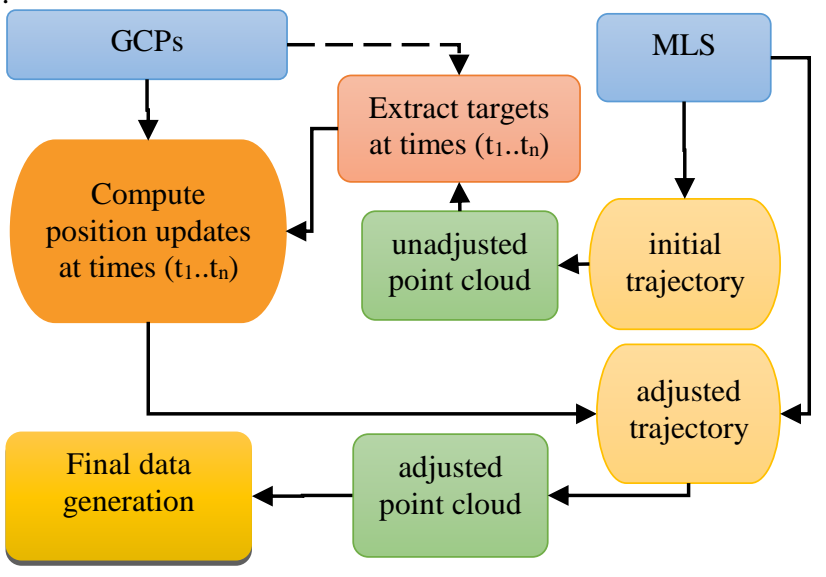

Figure 1: Schematic representation of trajectory adjustment procedure

\section{VALIDATION OF CONCEPT}

\subsection{Test setup}

In the frame of an airborne and mobile data acquisition campaign near Martigny (Switzerland) a more than $5 \mathrm{~km}$ long section with tunnels and galleries (from Bourg St. Pierre to the north entry of the Great St. Bernhard tunnel) was acquired by mobile mapping in September 2013. The section was acquired in in both directions driving at $40-50 \mathrm{~km} / \mathrm{h}$, thus recovering a GNSS signal on each side after approximately 6 minutes of driving.

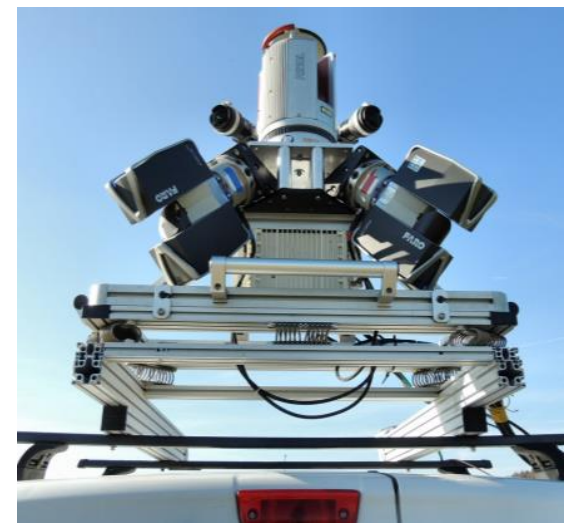

Figure 1: Mobile mapping system IGI-SAM used for the test in the tunnel section

The measures were performed using the IGI-SAM system that is composed of the following elements (see figure 2):

Two scanners Faro Focus 3D (scanning rate up to $250^{\prime} 000 \mathrm{pt} / \mathrm{sec}$ ) tilted by $45^{\circ}$ both in roll and pitch in order to measure a full $360^{\circ}$ profile of any road section and also any perpendicular faces of road infrastructure

One scanner Riegl VZ400 (scanning rate up to $150^{\prime} 000 \mathrm{pt} / \mathrm{sec}$ ) to scan the soft shoulder of the road 

purposes

Two cameras 8Mpix for documentation

L1/L2 GNSS receiver and a navigation grade IMU (IGI IMU-IIe: FOG Bias $0.03^{\circ} / \mathrm{h}$ ) yielding an integrated position accuracy of $0.05 \mathrm{~m}$, roll $/$ pitch accuracy of $0.003^{\circ}$ and a heading accuracy of $0.007^{\circ}$ (Minten, 2009).

Within the tunnel section GCPs were measured by a line traverse approximately every $200 \mathrm{~m}$ with an accuracy of $1 \mathrm{~cm}$ in 3D. The points were materialized as circular white targets with a diameter of $30 \mathrm{~cm}$ (see figure $3 \mathrm{a}$ ). Following the procedure described in paragraph 2.2 , first the unadjusted point cloud was computed (figure $3 \mathrm{~b}$ ), then the circular targets were detected using the intensity values and the $3 \mathrm{D}$ displacement vectors to the GCP coordinates computed (figure 3c). Finally the timestamp of the laser point closest to the target center was extracted. These values were used to generate external observations to be inserted into to the Kalman Filter. The latter described steps were performed semi-automatically by a tool specially developed for this purpose by the contractors (partly shown in figure $3 \mathrm{c}$ ).

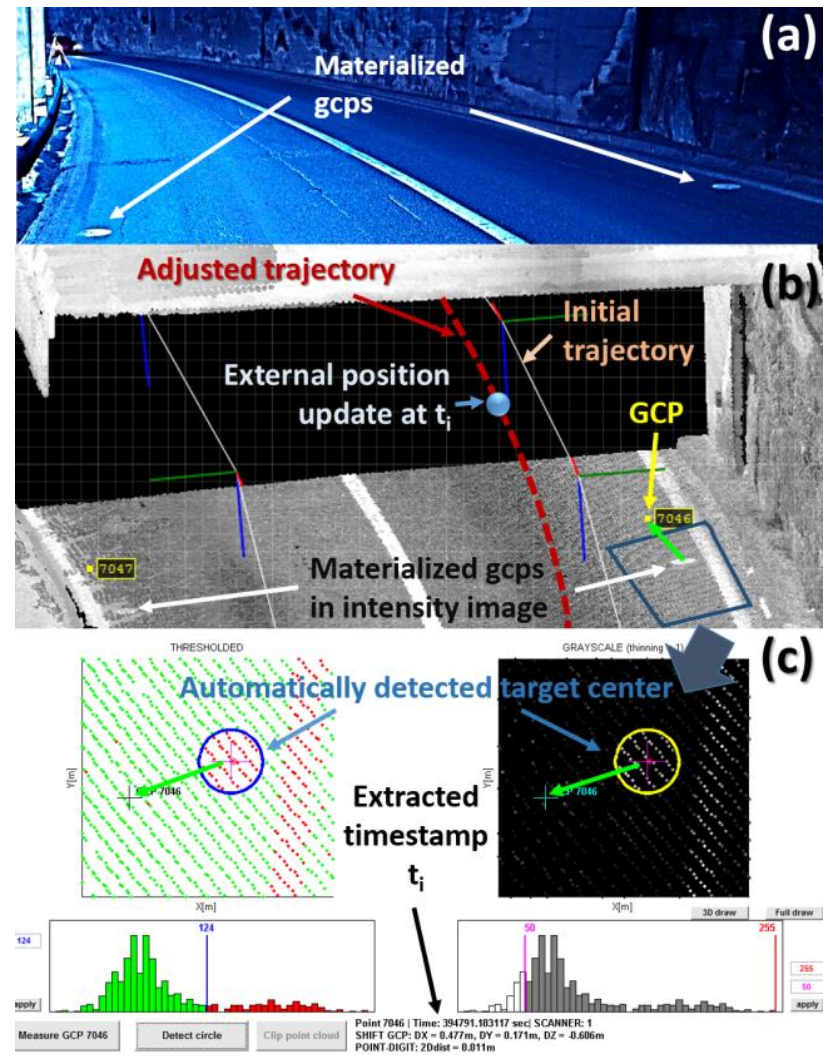

Figure 3: (a) Measure and materialization of targets with circular spots, (b) Initial point cloud color-coded by intensity, (c) Tool for detection of target centers, displacement vectors and timestamp extraction

\subsection{Results}

To evaluate what external update rate is required in order to reach a desired accuracy $(5 \mathrm{~cm}$ in this case), the computation of the trajectory and point cloud was performed using no GCPS (figure 4), GCPS every $1000 \mathrm{~m}$ (figure 5) and GCPS every $400 \mathrm{~m}$ (figure 6). For all scenarios, the target coordinate differences (in $\mathrm{XY}$ and $\mathrm{Z}$ ) were computed for all GCPs available.

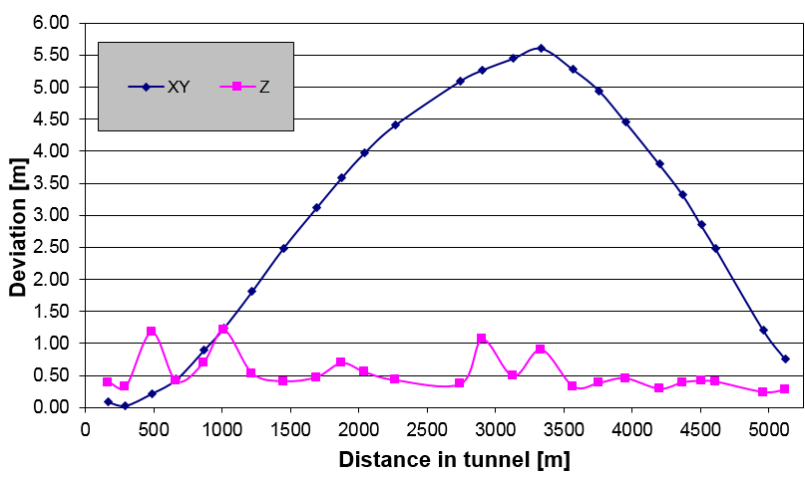

Figure 4: Deviation on GCPs without any adjustment

Deviation on GCP - 1GCP/1000m in KF

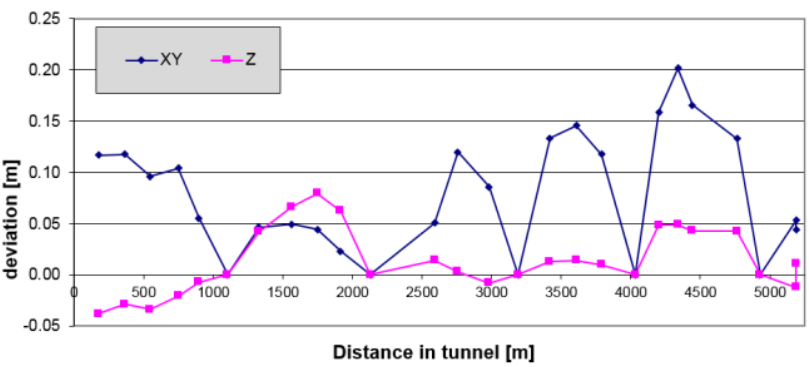

Figure 5: Deviation on GCPs using GCPS every $1000 \mathrm{~m}$ for KF update

Deviation on GCP - 1 GCP/400m in KF

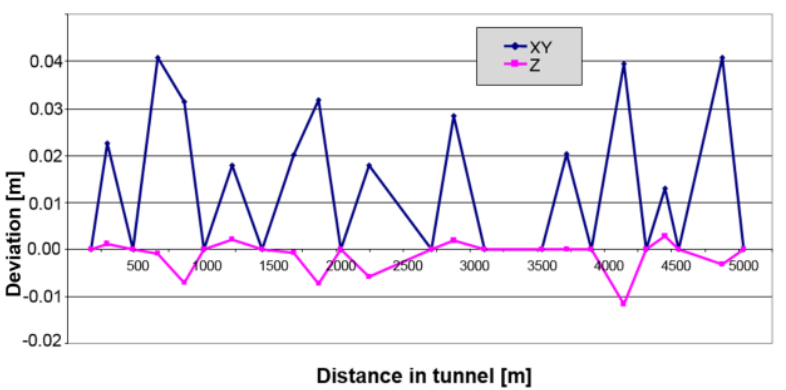

Figure 6: Deviation on GCPs using GCPS every 400m for KF update

The plot in figure 4 (pure inertial navigation for 6mins) shows that the position drift reaches its maximum $(5 \mathrm{~m}$ in $\mathrm{XY}$ and $1.2 \mathrm{~m}$ in $\mathrm{Z}$ ) approximately in the middle of the section. If the GNSS outage time is reduced by a factor 5 (update every $70 \mathrm{sec}$ ), the maximum observed position drift does not exceed $20 \mathrm{~cm}$ in $X Y$ and $7 \mathrm{~cm}$ in $\mathrm{Z}$ (figure 5) thus improving the position accuracy by a factor of 25. If the GNSS outage time is reduced even further (update every $400 \mathrm{~m} / 35 \mathrm{sec}$ ), the position accuracy can improved by a factor of $100(\max 5 \mathrm{~cm}$ in $\mathrm{XY}$ and $1 \mathrm{~cm}$ in $\mathrm{Z})$, approaching the integrated position accuracy without GNSS outages (figure 6). The measures show that the absolute position accuracy degrades at the square-root of the GNSS outage time. This reflects the fact that in inertial navigation position estimates are computed from accelerometer observations and that errors which arise in the accelerometers propagate through the double integration (Woodman, 2007).

\subsection{Interpretation of results}

Figure 7 shows the trend curve for the position drift in function of the GNSS outage for the XY and $\mathrm{Z}$ component. It can be stated that for the category of IMU used (navigation grade 
FOG) an external update every $\sim 30$ seconds (or every $400 \mathrm{~m}$ at $40-50 \mathrm{~km} / \mathrm{h}$ ) is enough to guarantee an absolute positioning better than $5 \mathrm{~cm}$ in $\mathrm{XY}$ and $3 \mathrm{~cm}$ in $\mathrm{Z}$. This allows to reduce drastically the number of target points that have to be measured in comparison to the method where the point cloud is adjusted a posteriori (no correction of the trajectory). However, if the MLS is equipped with a less performant IMU (tactical grade IMU), the update rates must probably be increased.

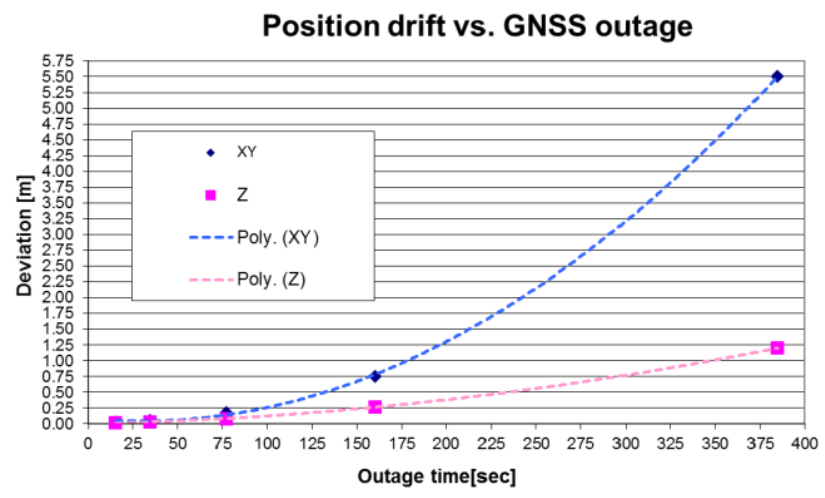

Figure 7: Computed trend curve for position drift in function of GNSS outage time for a navigation grade IMU

\section{CONCLUSION}

In this paper we have presented a methodology capable of delivering MLS data with absolute positioning better than $5 \mathrm{~cm}$ in $\mathrm{XY}$ and $3 \mathrm{~cm}$ even in long tunnels. The methodology consists in measuring targets as ground control points (GCPS), identifying the $3 \mathrm{D}$ point closest to the target's center and computing the coordinate difference to the corresponding GCP. By retrieving the timestamp of the closest laser measurement, an external position update at a given time can be computed. In comparison to an a posteriori point cloud adjustment, the usage of GCPS to generate external time-tagged observations for the KF allows to reduce the number of GCPs by a factor of 5 to 10 . The proposed methodology requires that the trajectory and the point cloud have to be computed twice, but the gain in accuracy and the economy in time on the field (less GCPs needed) clearly counterbalances this inconvenient. Additionally, if the GCPs are materialized by clearly identifiable patterns in the intensity color-coded point cloud (e.g. circular patterns), the target detection and external update computation can be highly automated.

\section{REFERENCES}

Baltsavias, E.P., 1999. Airborne laser scanning: basic relations and formulas. ISPRS Journal of Photogrammetry and Remote Sensing, 54(2-3): 199-214.

Minten, H. 2009. The Modular System Concept of IGI. In: FRITSCH, D. (Ed.): Photogrammetric Week '09, Wichmann, pp. 41-47.

Woodman J, 2007. An Introduction to Inertial Navigation ,Technical Report Number 696, Computer Laboratory UCAM-CL-TR-696 ISSN 1476-2986 